\title{
A Mobile Application for Reporting Disease Incidents
}

\author{
Hylke van der Schaaf ${ }^{1}$, Ente Rood ${ }^{2}$, and Kym Watson ${ }^{1}$ \\ 1 Fraunhofer IOSB, Frauhoferstraße 1, 76131 Karlsruhe, Germany \\ \{hylke.vanderschaaf, kym. watson\}@iosb.frauhofer.de \\ 2 Koninklijk Instituut voor de Tropen, Amsterdam, The Netherlands \\ E.Rood@kit.nl
}

\begin{abstract}
The current procedure for the reporting of cholera cases in Uganda contains many manual steps across several levels of the health infrastructure. Because of this there is a large chance of errors in the information flow, possibly delaying the signalling of an outbreak. The lack of accurate and complete data also hinders research into the spread of cholera. To improve the cholera reporting an application called Dira (Disease Incidence Reporting Application) has been developed for mobile devices, that allows the field registration of patients to be done quickly, easily and accurately. By entering the data directly on an electronic device there is no longer the need for separate digitization steps. By transferring case data directly from the hand-held device to a central server at the Ugandan Ministry of Health, the reliability of the data can be increased and the time to launch a response to an outbreak can be decreased.
\end{abstract}

Keywords: cholera,health,reporting,mobile application.

\section{Introduction}

EO2HEAVEN (Earth Observation and ENVironmental modelling for the mitigation of HEAlth risks, EU FP7 244100, [1]) has developed methodologies, correlation models, spatial data services (using OGC standards) and applications supporting the main activities in environmental health. EO2HEAVEN work is organized in three case studies: a) environmental effects on allergies and cardiovascular diseases in Saxony, Germany, b) environmental challenges to health caused by air pollution from petrochemical plants in Durban, South Africa, and c) investigating the impact of climate variables on cholera outbreaks in Uganda.

The research in the cholera case study requires accurate records of cholera cases. Currently, cases of cholera are recorded on paper at the cholera treatment centres. These forms are then digitized and sent, once a week, by email and hard-copy, to the District Health Office. The District Health Office gathers the reports of the district, digitizes them if needed, and sends the aggregated data to the Ministry of Health. This whole process takes up to two weeks, hindering a timely response to a cholera outbreak. The process also involves many manual

J. Hřebíček et al. (Eds.): ISESS 2013, IFIP AICT 413, pp. 188-195, 2013.

(C) IFIP International Federation for Information Processing 2013 
copy actions, making it very error-prone and making the resulting cholera case data unreliable. Since a patient record, once sent, can not be updated the cholera case data available at the Ugandan Ministry of Health is often incomplete or erroneous.

To improve the cholera reporting in Uganda, an application called Dira (Disease Incidence Reporting Application) has been developed for mobile devices, that allows the field registration of patients to be done quickly, easily and accurately. By entering the data directly on an electronic device there is no longer the need for separate digitization steps, while by transferring case data directly from the hand-held device to a central server at the Ministry of Health, the reliability of the data can be greatly increased and the time to launch a response to an outbreak can be greatly decreased.

\section{Health Surveillance and Health Information Management}

Public health surveillance aims at the collection, analysis, interpretation, use and dissemination of data for guiding decision making to prevent and control the occurrence of disease and injury [6]. The fundamental principle of such a system is to provide valid, accurate, unbiased and timely data to decision makers to plan interventions. As the process of collecting detailed health information can become very cost inefficient when conducted on a small temporal and spatial scale and will compromise the quality of the data collected, most surveillance systems rely on aggregated data collated at district levels and reported monthly to national authorities [5]. The utility of surveillance data, collected on various time intervals, can serve various objectives for the assessment of and the response to the occurrence of disease. The immediate detection of emerging outbreaks, for example, will rely on a prompt and timely reporting of newly detected cases to monitor the progression of an epidemic and to monitor the effectiveness of instantaneous interventions. At the same time, health system monitoring and resource allocation in National health policies will require quality data available over more extended time periods.

\section{Cholera Surveillance and Reporting in Uganda}

In 2000 the Ugandan ministry of Health adapted the Integrated Disease Surveillance and Response (IDSR) strategy with the intention to achieve an integrated and intervention oriented surveillance system as outlined in the International Health Regulations [8]. This framework uses various surveillance indicators (core and process) to monitor trends in the occurrence of communicable diseases and to evaluate the effectiveness of program interventions [4]. The IDSR integrates the systemic collection and dissemination of a number of diseases, previously monitored by different vertical disease programs across all layers of the health care system, including cholera. The core functions of this system are to 1) detect 
cases in a timely fashion, 2) record cases according to quality standards, 3) to report cases transparently using an adequate communication network and 4) to analyze the available data for response planning and epidemic mitigation [5].

An evaluation of the IDSR system functions conducted in 2008, showed a considerable improvement of surveillance system functions over the period 2000-2007 4. Both the completeness of reports as well as the timeliness of monthly reporting increased substantially (respective increase over period 2001-2007 of report completeness: $49 \%-85 \%$; and timeliness: $50 \%-77 \%)$. In contrast to the increase in monthly reports sent to the National surveillance department, the consistency of weekly reports submitted showed a short upsurge from $49 \%$ in 2001 , to $96 \%$ in 2004 , but diminished again to $53 \%$ by the year 2007. The authors conclude that this reduction can be attributed to reduced financial investments by the National government leading to reporting gaps.

A descriptive assessment of the cholera surveillance system in Uganda, conducted in the Kasese district in April 2012 as part of the EO2HEAVEN project (unpublished data) showed several quality measures in place including the confirmation of the completeness and timeliness of reports. However, at this point, limited effort had been invested to perform regular data quality checks or further data analysis within the district. Data validation of reports is a core activity by the Ministry of Health though this is not consistently

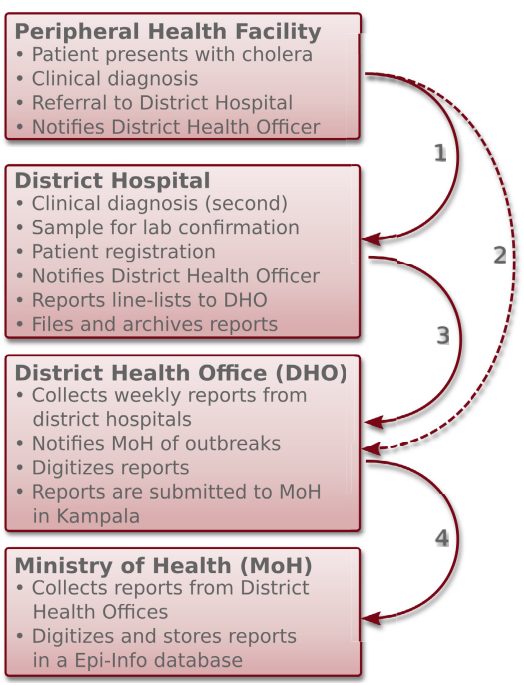

Fig. 1. Current cholera reporting procedure: 1 .New cholera cases are directly reported from the peripheral health centers to the district health office. 2.Weekly cholera reports are sent to the DHO to trace epidemic outbreaks. 3.New Cholera cases are directly reported from district health office to the MoH. 4.Monthly reports are compiled at the $\mathrm{DHO}$ and sent to the $\mathrm{MoH}$ to monitor cholera prevalence. $5 . \mathrm{MoH}$ reports to the WHO and other international initiatives (not shown). done due to inadequate resources. Post outbreak evaluation is standard practice that the Ministry of Health recommends for all affected districts but this too is subject to availability of resources to conduct the evaluation [3].

The current data flow and digitization process has not been standardized across different levels of the health infrastructure. Capacity to digitize information and to perform basic data analysis or visualization tasks is limited. Likewise the current staff lacks basic knowledge of data management and analysis. Even though weekly reports and time series graphs are produced from the data, the lack of data quality checks and critical evaluation of the data limits the efforts 
to interpret the results. As a result, data managers have little to no sense of ownership and perceived value of their work for others, resulting in a limited accountability. The stratification of the health information system over multiple administrative levels confines the prompt dissemination of information. This also introduces errors in the information flow, potentially delaying the signalling of an outbreak when it occurs. Even though alternative procedures exist to flag initial cases for follow up investigations, the protocols are fuzzy and the system is prone to human error. Moreover the lack of resources limits the efforts for efficient active surveillance. It appears that the flow of information from the peripheral health facilities to the ministry of health is hampered by lack of tools to collect, store and disseminate this information. The reporting system relies on various consecutive steps as visualized in Fig. 1]

Based on the requirements identified, EO2HEAVEN has developed a standardized data collection protocol and associated data collection tool to facilitate data collection and provide access to health information across all levels of the health infrastructure. The tool will benefit both health practitioners working in the field as well as health authorities working at the national ministry of health as it 1) Reduces the number of human errors induced by the current reporting system 2) Reduces the amount of resources spent on data management and dissemination 3) Improves response time, 4) Increases the effectiveness of surveillance by facilitating the exchange and interpretation of information at a sub-district level and 5) Facilitates communication across all levels of the health system.

\section{Dira: A Mobile Application for Incident Reporting}

Dira, Disease Incidence Reporting Application is an application for tablet PCs and smartphones running the Android platform. It is developed to improve the accuracy and speed of recording of cholera cases in third world countries. The main screen (see Fig. 2) of the application is the listing of the entered cases. From here cases can be directly viewed and searched. All other functionality is directly available from the menu.

\subsection{Data Entry}

The application has an easy-to-use interface for entering patient data. It allows for the collection of primary patient data such as name and home town, symptoms and epidemiological information, such as the patients primary water source, and which villages he or she has visited in the week before falling ill.

To increase the ease of entering village names, to reduce variations in spelling of village names, and to insure that the correct village is entered when multiple villages with the same name exist in different counties, the application has a database of village names, sorted by sub-county, county and district. When entering a village name, the user is prompted for the district, county, sub-county and village name. If the user is certain of the spelling of the name of the village, he can directly type in the village name. The system will give as-you-type 


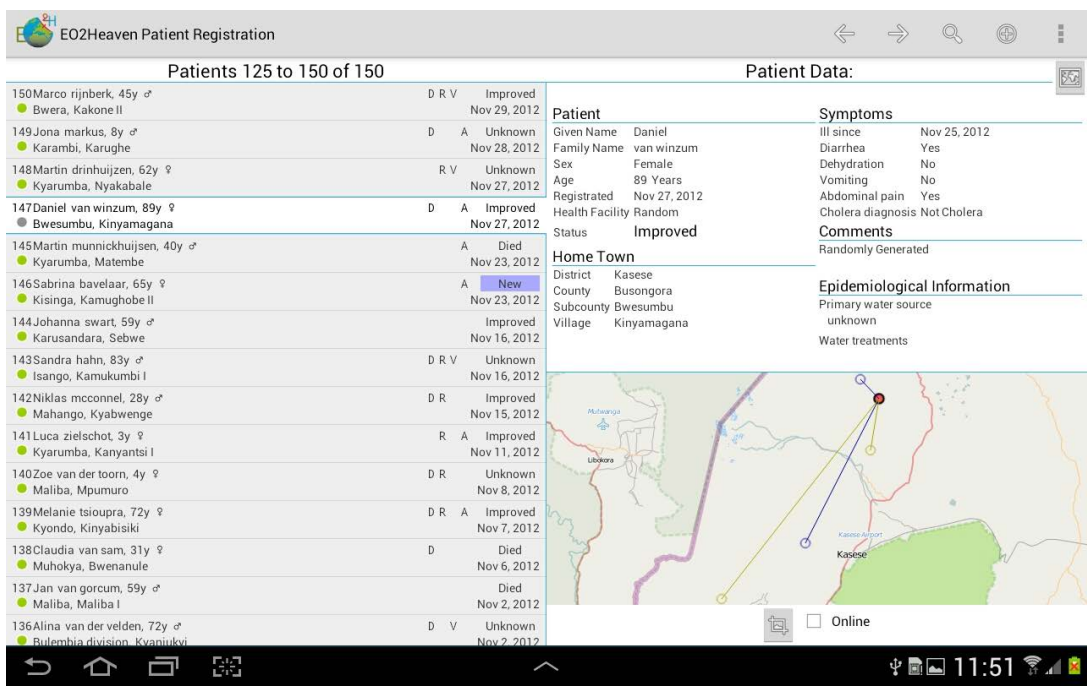

Fig. 2. Dira main screen

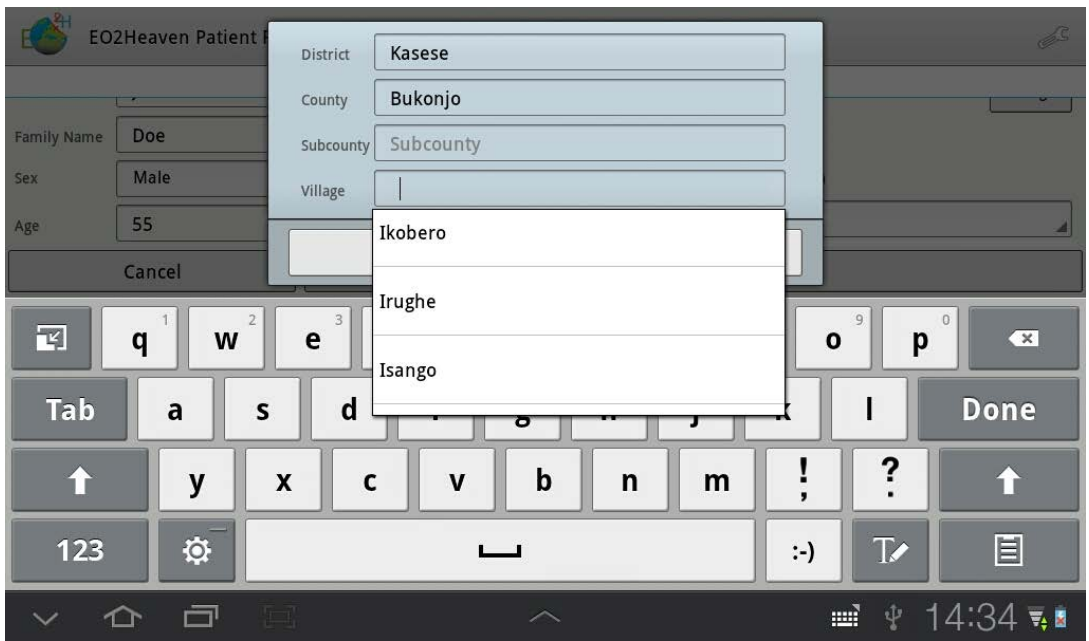

Fig. 3. Entering a village name

suggestions based on all known villages. When the user selects one of these suggestions, the district, county and sub-county names will be automatically filled in. If the user is not certain of the village name, he can drill-down by starting with the district, county or sub-county. Once one of these fields is filled in, the suggestions for the other fields will be limited to entries matching the already completed fields (see Fig. 3). The user can always override the suggestions by typing in the full village name. 


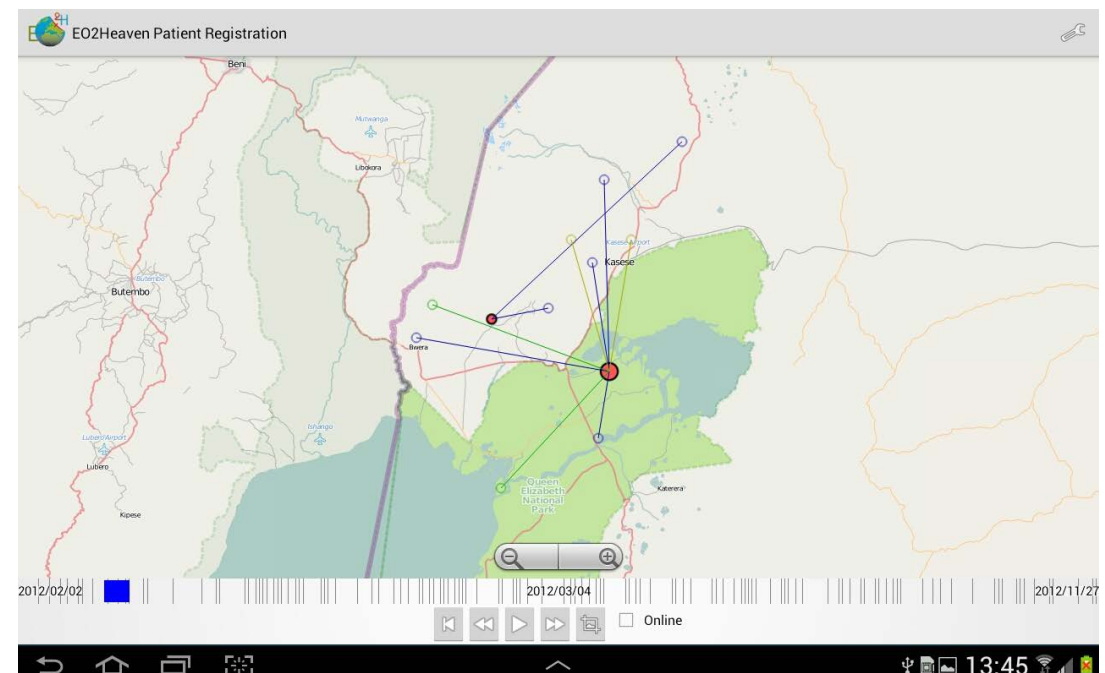

Fig. 4. Display of cases on a map, animated in time

When editing a case, before the changes are stored, the user is presented with an overview page highlighting all changed fields in the patient record. This inbetween page allows the user to confirm that he did not make any accidental changes while working with the input form.

\subsection{Data Visualisation}

The application has several ways to visualize the data. It can show graphs of cases aggregated per week, for several time periods (running or previous month, last 6 or 12 months, all). It can show individual cases on a map, together with the villages that the patient has visited in the week before he became ill. It can also show all, or a subset of cases on a map, animated in time, giving a visual overview of the progress of an outbreak (see Fig. 4).

\subsection{Data Transmission}

To gather the data in a central place the application can send the patient data to a central server over any available internet connection, e.g. Wi-Fi or GSM. The application has been made robust for field work with intermittent internet connectivity. If the data transfer is interrupted, the application will automatically resend the data from the point of failure. The application will also automatically resend patient records that have been updated, meaning patient status can be updated even after the patient record has been sent to the central server, leading to a more complete data set. 


\subsection{Data Output}

It is important for the uptake of the application that its use can seamlessly integrate into the existing workflows at the local level, with respect to data collection and archiving. To facilitate this, the application can export the data records available on the device in various file formats, like CSV for importing into existing patient record systems, and PDF for easy printing.

Since internet connectivity is not always available in Uganda, these exports will still allow the old case-reporting methods to be used, by sending printouts from the local treatment centres through the District Health Offices, to the Ministry of Health. This will give the users the confidence that even if there is no internet connectivity, their data-collecting efforts are not in vain.

\subsection{Implementation Details}

The platform used to implement Dira is Android. The choice for Android was made because of the large diversity of devices available with this platform, from low-cost phones to high-performance tablets. Some ebook-readers with an e-ink display also run Android and these e-readers could be very suitable for data collection in the field, as their screens do not become hard to read in very bright sunlight, and they use very little power. We have not yet been able to test any e-readers.

For field work it seems that 7 inch tablets are optimal. Devices of this size are easy to hold in one hand, which is important when used while standing or walking, yet they are still large enough to give a good overview when using forms or maps.

The application is implemented as a native Android application, making it very resource efficient. This also allows it to fully utilize the internationalization functionality of the platform and gives it the ability to easily specify different layouts for different device sizes and portrait/landscape orientations. For storing patient data on the device, the OS-provided SQLite database is used. This database also stores village data that is used to give autocomplete / as-you-type suggestions when entering a patients home town or visited locations. For graphing functions are implemented with the AchartEngine library [7. For the map display functions, the Mapsforge library [2] is used, which can work with off-line vector maps. This allows the map functionality to work fully without internet connectivity.

\section{Conclusions and Recommendations}

The Dira application was received very well by local health workers when they tried it out at two EO2HEAVEN workshops in Uganda. Users especially appreciated the ease of use of the application. A key feature is its ability to work completely off-line with no internet access. Feedback from these workshops will be used to further improve the application. It is important that the application 
is smoothly integrated in the current organisational workflows. The vision is to incorporate this application into an early warning system for different diseases in developing countries.

Acknowledgments. The research leading to these results has received funding from the European Community's Seventh Framework Programme (FP7/20072013) under grant agreement $n^{\circ} 244100$ (EO2HEAVEN)

\section{References}

1. EO2HEAVEN: Earth observation and environmental modelling for the mitigation of health risks (june 2013), http://eo2heaven.org/

2. mapsforge: free mapping and navigation tools (june 2013), http://code.google.com/p/mapsforge/

3. Lukwago, L.: personal communication (February 2012)

4. Lukwago, L., Nanyunja, M., Ndayimirije, N., Wamala, J., Malimbo, M., Mbabazi, W., Gasasira, A., Nabukenya, I.N., Musenero, M., Alemu, W., Perry, H., Nsubuga, P., Talisuna, A.: The implementation of integrated disease surveillance and response in uganda: a review of progress and challenges between 2001 and 2007. Health Policy and Planning 28, 30-40 (2013)

5. Nsubuga, P., White, M.E., Thacker, S.B., Anderson, M.A., Blount, S.B., Broome, C.V., Chiller, T.M., Espitia, V., Imtiaz, R., Sosin, D., Stroup, D.F., Tauxe, R.V., Vijayaraghavan, M., Trostle, M.: Disease Control Priorities in Developing Countries chap. Public Health Surveillance: A Tool for Targeting and Monitoring Interventions, pp. 997-1015. World Bank, Washington (2006)

6. Thacker, S., Berkelman, R.: Public health surveillance in the united states. Epidemiol Rev. 37, 1-18 (1988)

7. The 4ViewSoft Company: AChartEngine (june 2013), http://www . achartengine.org

8. WHO: International Health Regulations (2005). WHO (2008) 